OPEN

SUBJECT AREAS:

DRUG DEVELOPMENT

BIOMATERIALS - CELLS

NANOSCALE BIOPHYSICS

NANOPARTICLES

Received

10 April 2013

Accepted

7 May 2013

Published

17 June 2013

Correspondence and requests for materials should be addressed to S.J.L. (sjlee@postech. ac.kr)

\section{Controlled cellular uptake and drug efficacy of nanotherapeutics}

\author{
Sungsook Ahn' ${ }^{1}$ Eunseok Seo ${ }^{2}$, Kihean Kim ${ }^{2,3}$ \& Sang Joon Lee ${ }^{1,2,3}$
}

\begin{abstract}
${ }^{1}$ Biofluid and Biomimic Research Center, ${ }^{2}$ Division of Integrative Biosciences and Biotechnology, ${ }^{3}$ Department of Mechanical Engineering, Pohang University of Science and Technology, Pohang, 790-784, Korea.
\end{abstract}

Cellular uptake pathway of nanoparticle (NP) is different from that of free drugs. Therefore, NP-mediated nanotherapeutics can be designed to overcome the adverse effects of free drugs. However, synthetic NPs are typically trapped in the endosome and have difficulty to reach the cytosol because of the characteristic endocytosis, where the endosomal membranes wrap-up the introduced NPs. In this study, the Spacer molecules linking the apoptotic anticancer drug and the gold NP (AuNP) are designed and cellular uptake procedure and drug deployment in the cancer cells are controlled. X-ray nanoscopy and two-photon microscopy are employed to observe the AuNPs in a cell in-situ without additional dye molecule or imaging agent introduction on an AuNP. We confirm that the effective design of the Spacer molecules importantly control the cellular interaction of the AuNPs. This technology can be generalized to broad biomedical applications utilizing nanotherapeutics-mediated diagnosis and new-concepted disease treatment technologies.

N anotherapeutics commonly utilizes small-molecule drugs, peptides, proteins, and nucleic acids loaded in nano-scale carriers such as nanoparticle (NP) made of organic polymers, inorganic particles, biological lipids, and so on. NPs are uptaken into a cell known as endocytosis procedure, while free drugs typically pass through the membrane unless they are kicked out of the cell by efflux pump mechanism ${ }^{1,2}$. Enhanced cellular uptake and transport of NPs through the cell membrane driven by synthetic or biologically-modified molecules is of intense interest ${ }^{3}$. Some natural materials such as cell-penetrating or cell-fusogenic peptides promote cell penetration efficiently ${ }^{4,5}$. However, synthetic NPs are typically trapped in the endosome because of that endocytosis procedure by which sub-membranes are formed. This procedure thus inherently inhibits deep penetration of NPs into the cytosol ${ }^{6,7}$. Nonetheless, since the cellular uptake pathway of NP is different from that of free drugs, NP-mediated nanotherapeutics has a great potential to overcome the adverse effects of free drugs such as multidrug-resistance or undiscerning-selectivity to cancers, and so on $^{8}$. With improved pharmacokinetics and pharmacodynamics, the therapeutics loaded in NP can be safer and more effective compared with free drugs ${ }^{9}$. In addition, passive tumor-targeting naturally utilizes the irregularity and the leakiness of tumor vasculature, which is beneficial for NP accumulation in the tumor area (enhanced permeation and retention effect) ${ }^{10,11}$. However it still remains unknown how NPs move through tumor tissues once they are localized in a tumor area and how they carry the drug molecules into tumor cells before releasing ${ }^{12}$. Therefore, modification of endocytosis procedure and single-cell level tracking of the nanotherapeutics are fundamentally needed.

NPs enter cells through endocytosis: phagocytosis for the uptake of large particles and pinocytosis for fluids and solutes ${ }^{13,14}$. Since NPs have a high surface-to-volume ratio due to their small size, effective control of NP surface is also critical for targeted applications ${ }^{15-17}$. A neutral ligand such as poly(ethylene glycol) (PEG) resist protein adsorption and reduce nonspecific endocytosis uptake $e^{18,19}$. On the other hand, cationic particles advantageously bind to anionic functional groups on a cell surface (e.g., sialic acid) ${ }^{20}$ and translocate across the plasma membrane through a clathrin-mediated uptake pathway ${ }^{21}$. Then, neutral and negatively charged NPs are expected to be less efficiently adsorbed and uptaken by a negatively charged cell membrane ${ }^{22}$. However, the cellular uptake of negatively charged NPs is also high through strong and nonspecific interactions with the plasma membrane ${ }^{23-27}$. Concentration and spatial configuration of the ligand molecules ${ }^{13}$, charge density ${ }^{14}$ and additional functional group introduction ${ }^{28}$ on a NP surface affect the cellular uptake processes. Therefore, cellular interaction of NP is not that simple enough to set-up the universal rules.

Here, we develop characteristic Spacer molecules binding anticancer drug molecules on a gold NP (AuNP) and investigate cellular uptake efficiency and spatial distributions of AuNPs in a cancer cell. Due to high X-ray absorption ability and unique photo-physical properties of Au element, AuNPs in a cell are tracked by X-ray imaging and wavelength-controlled two photon microscopy without additional dye molecule or imaging agent 
introduction. By this way, cellular interaction of molecules introduced on an AuNP is effectively compared without complication by additional molecular interactions. Single-cell level in-vitro analysis of drug-conjugated AuNPs is investigated in terms of incorporation efficiency and spatial distribution in a cell according to the designed Spacer molecules. The in-vitro drug efficiency is extended to in-vivo tumor model to elucidate the drug efficacy relation in a cell and collective tumor tissues.

\section{Results}

Spacer molecule design for anticancer drug (Doxorubicin, DOX) - conjugated AuNPs. To investigate cellular uptake behavior and drug efficacy of NP therapeutics in a cell, we design five thiol-linked Spacers I to V (Fig. 1a) tethering apoptotic anticancer drug, Doxorubicin (DOX) on the surface of the negatively-charged AuNP 1 and positively-charged AuNP 2 (Fig. 1b), respectively. The chemical structure of the spacer molecules linked to the anticancer drug is displayed in Fig. 1a. The amine group of DOX is linked to the carboxyl group of each spacer molecule, forming a peptide linkage. Spacer I is bulky and hydrophobic, whereas Spacer II is linear and hydrophobic. Spacers III and IV are composed of hydrophilic PEG molecules with different molecular weights. Spacer V is amphiphilically composed of hydrophobic Spacers II and hydrophilic Spacer III in a $1: 1$ molar ratio. For all the designed AuNPs, the number of DOX-tethered spacer on each AuNP is controlled at approximately $1 \times 10^{3} \mathrm{DOX} /$ AuNP (Supporting Information). After the DOXtethered spacer is attached, the remaining active sites on the
AuNP surface are occupied by charged molecules with anionic (- $\mathrm{CH}_{2} \mathrm{COOH}$, AuNP 1 series) and cationic $\left(-\mathrm{C}_{5} \mathrm{H}_{4} \mathrm{~N}_{5}\right.$, AuNP 2 series) functionalities as summarized in Fig. 1b. The surface charge of AuNP is beneficial for adsorption onto the cell, while the designed Spacers are expected to modify the pathways through which NPs penetrate the cell membrane bringing DOX into a cell.

Four control systems and ten designed AuNPs are compared in Figure 2. The control systems include free DOX, charge-only AuNP 1 and AuNP 2, as well as PEG 5000-only AuNP. Free DOX and PEG 5000-only AuNPs have no apparent charge, whereas AuNP 1 and AuNP 2 exhibit prominent charge. The $\zeta$-potential of DOX-tethered AuNPs become partially neutralized, except Spacer I. The degree of DOX-tethered Spacer introduction on an AuNP may not be directly proportional to the $\zeta$-potential change, but rather affect the physical properties including the electrophoretic mobility of the AuNPs and hydrodynamic properties. The surface-modification of each AuNP and drug loading efficiency is confirmed by fluorescence and UV-vis spectrum (Supporting information, Fig. S1).

AuNP incorporation into a cancer cell. The amount of AuNPs incorporated in adenocarcinomic human alveolar basal epithelial cancer cell (A549) is quantitatively analyzed at the designed time points using energy dispersive X-ray spectroscopy (EDXS) combined with scanning electron microscopy (SEM). The representative EDXS results are provided in Fig. S2 and typical SEM images of each AuNP-incorporated system are provided in Fig. S3. The typical proliferation time for A549 is approximately $24 \mathrm{~h}$. Therefore,

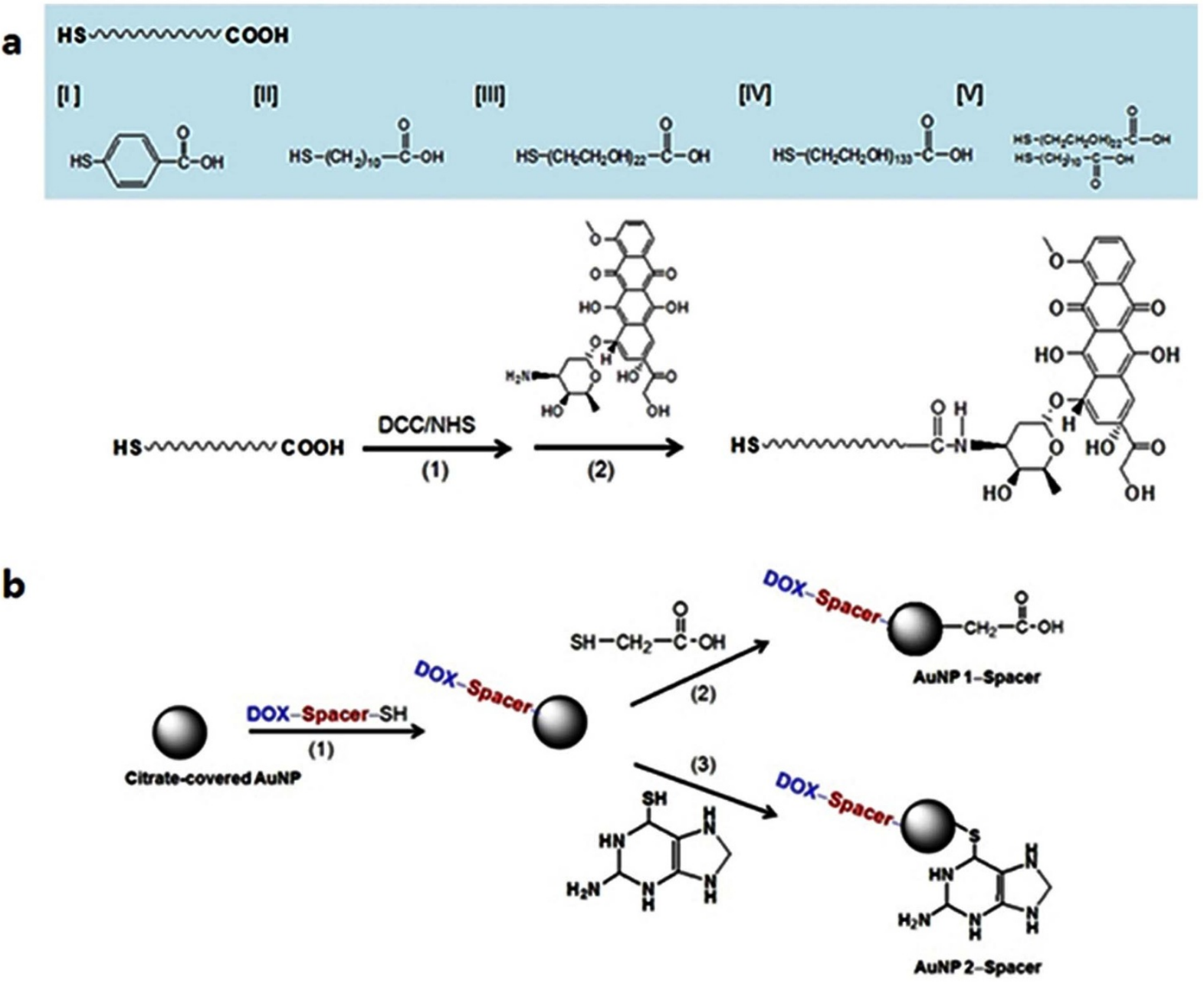

Figure $1 \mid$ (a) Synthetic procedure of the Spacer molecules. (b) Synthetic procedure of the drug-conjugated and surface-modified AuNPs. 


\begin{tabular}{|c|c|c|c|c|c|}
\hline & Surface-modification & Spacer Moiecuie & $\begin{array}{l}\mathrm{D}_{\mathrm{H}^{[1]}} \\
(\mathrm{nm})\end{array}$ & $\begin{array}{l}\text { C-Potential[ }{ }^{[l]} \\
\quad(\mathrm{mV})\end{array}$ & $\begin{array}{c}\text { Max. Au contents } \\
\text { in a cell (wt \%) } \\
\text { \& time (min) }\end{array}$ \\
\hline Doxorubicin & $\mathrm{N} / \mathrm{A}$ & $\mathbf{N} / \mathbf{A}$ & $\mathrm{N} / \mathrm{A}$ & 0.025 & N/A \\
\hline AuNP1 & $-\mathrm{S}-\mathrm{CH}_{2} \mathrm{COOH}$ & $\mathrm{N} / \mathrm{A}$ & 45 & -25 & $28.01(72 \mathrm{hr})^{[2}$ \\
\hline AuNP2 & $-\mathrm{S}-\mathrm{C}_{5} \mathrm{H}_{4} \mathrm{~N}_{5}$ & $\mathrm{~N} / \mathrm{A}$ & 56 & 31 & $30.58(3 \mathrm{hr})^{[2]}$ \\
\hline AuNP-PEG 5000 & -S-PEG 5000 & N/A & 139 & -0.019 & N/A \\
\hline AuNP1-I & & & 3078 & 0.081 & $75.91(180 \mathrm{~min})$ \\
\hline AuNP1-II & & $-\mathrm{S}-\left(\mathrm{CH}_{2}\right)_{10}-\stackrel{\mathrm{O}}{\mathrm{C}} \cdot \overrightarrow{\mathrm{N}}-\mathrm{N}-\mathrm{DOX}$ & 1085 & -11.26 & $45.28(40 \mathrm{~min})$ \\
\hline AuNP1-III & & 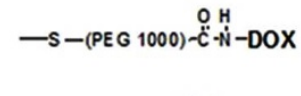 & 157 & -14.24 & $5.33(40 \mathrm{~min})$ \\
\hline AuNP1-IV & & 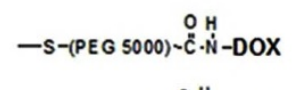 & 193 & -16.20 & $8.91(120 \mathrm{~min})$ \\
\hline AuNP1-V & & $\begin{array}{c}\text {-S-(PEG 1000)-CH }-\mathrm{H}-\mathrm{N}-\mathrm{DOX} \\
-\mathrm{S}-\left(\mathrm{CH}_{2}\right)_{10}-\mathrm{C}-\mathrm{N}-\mathrm{DOX} \\
\mathrm{O} \\
\mathrm{H}\end{array}$ & 533 & -16.93 & $42.09(420 \mathrm{~min})$ \\
\hline AuNP2-I & & & 1086 & 3.91 & $52.32(180 \mathrm{~min})$ \\
\hline AuNP2-II & & $-s-\left(\mathrm{CH}_{2}\right)_{10}-\stackrel{O}{\mathrm{C}} \cdot \stackrel{\mathrm{H}}{\mathrm{N}}-\mathrm{DOX}$ & 753 & 14.24 & $32.13(120 \mathrm{~min})$ \\
\hline AuNP2-III & & -S-(PEG 1000)- $\tilde{C}^{-\stackrel{H}{*}-\mathrm{N}}-\mathrm{DOX}$ & 248 & 12.22 & $26.23(60 \mathrm{~min})$ \\
\hline AuNP2-IV & & 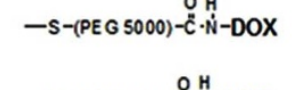 & 234 & 11.06 & $18.54(30 \mathrm{~min})$ \\
\hline AuNP2-V & & $\begin{array}{c}\text {-S-(PEG 1000)- }-\mathrm{H}-\mathrm{H}-\mathrm{DOX} \\
-\mathrm{S}-\left(\mathrm{CH}_{2}\right)_{10}-\mathrm{C}-\mathrm{N}-\mathrm{N}-\mathrm{DOX} \\
\mathrm{O} H \mathrm{H}\end{array}$ & 308 & 13.32 & $25.34(120 \mathrm{~min})$ \\
\hline
\end{tabular}

Figure $2 \mid$ Hydrodynamic diameter $\left(\mathrm{D}_{\mathrm{H}}\right)$ and zeta $(\zeta)$-potential of the AuNPs. ${ }^{[1]}$ Measured in de-ionized water at $25^{\circ} \mathrm{C}$. The concentration of the AuNP solution is controlled by $1 \mathrm{mM} \mathrm{Au} .{ }^{[2]}$ The maximum values for AuNP 1 and AuNP 2 are obtained with different time intervals from 3 to $72 \mathrm{hr}$ (Supporting Information, Fig. S4).

within the designed time points in this study $(<12 \mathrm{~h})$ and with the appropriate concentration of employed AuNP solution, the dilution of the AuNP into the divided cells is minimized. To compare cellular uptake efficiency of the designed AuNPs, the temporal variation of Au wt\% in all the designed systems is graphically displayed in Fig. 3. Each point in the graph is averaged from ten cases after excluding the maximum and the minimum with the deviation within $\pm 10 \%$, which is not shown on the graph for clear presentation. The timedependent maximum $\mathrm{Au} w \mathrm{wt} \%$ incorporated in a cell is summarized in Fig. 2 (also in Fig. S4 for charge only AuNP 1 and AuNP 2). The results exhibit characteristic patterns according to the Spacer type and charge, implying that the cellular interactions of the designed AuNPs are dominated by the Spacer molecules. The hydrophobic
Spacer is expected to be beneficial to pass through the membrane of a hydrophobic barrier. Au wt\% of AuNP 1-I and AuNP 2-I is maximized at $180 \mathrm{~min}$ and then gradually decreased. The maximum value of AuNP 1-I is higher than that of AuNP 2-I. AuNP 1-II and AuNP 2II also exhibit a maximum, where the maximum Au wt $\%$ of AuNP 1II is higher and faster than that of AuNP 2-II. Hydrophobic Spacers display maximum AuNP incorporation at a certain time. In that point, bulky hydrophobic Spacer I displays higher maximum at faster time than those of linearly hydrophobic Spacer II for both anionic AuNP 1 and cationic AuNP 2.

PEG-conjugated AuNP 1-III and AuNP 1-IV have relatively low $\mathrm{Au}$ wt $\%$ with some fluctuations. Despite these fluctuations, the maximum Au wt\% of the cationic AuNP 2 series is slightly higher than
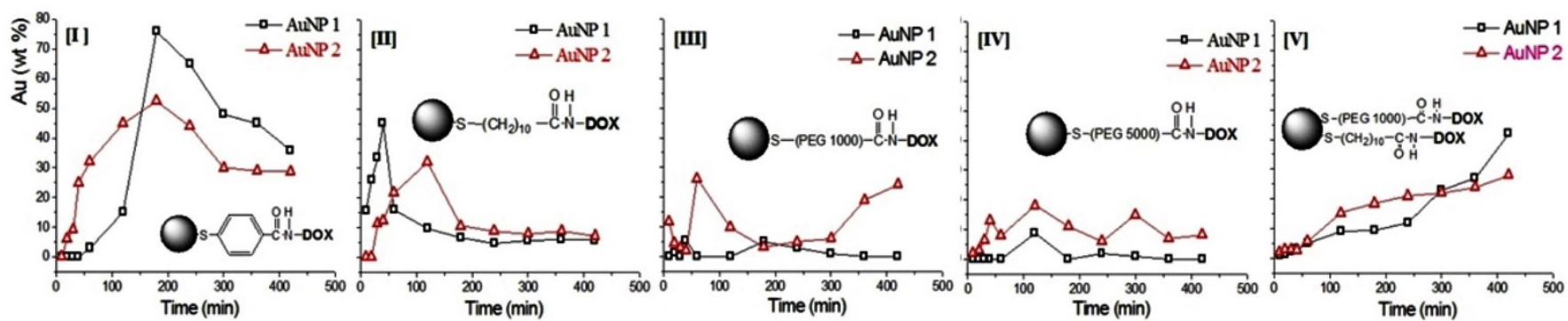

Figure 3 Time-dependent Au wt\% in A549 cells treated with the AuNPs having designed Spacers I, II, II, IV and V, combinated with cationic $\left(-\mathrm{CH}_{2} \mathrm{COOH}, \mathrm{AuNP} 1\right)$ and anionic $\left(-\mathrm{C}_{5} \mathrm{H}_{4} \mathrm{~N}_{5}, \mathrm{AuNP} 2\right)$ surface-modification. 
that of anionic AuNP 1 series, while the molecular weight effect of PEG is relatively negligible. The stealth function of PEG can inhibit cellular uptake, nonetheless effective interaction of cationic AuNP 2 with a cell membrane may help to obtain higher Au wt $\%$ in the AuNP 2 series over that of AuNP 1 series. Randomly fluctuating Au wt\% of the PEG-conjugated AuNPs implies that the AuNP-cell interaction is far from an energy-consuming endocytosis. On the other hand, amphiphilically combined Spacer $\mathrm{V}$ shows unique temporal variation different from the original Spacers II and III. Combined with either AuNP 1 or AuNP 2, amphiphilically combined Spacer V exhibits low Au wt $\%$ at the initial stage but continuous increase along with time. The increase rate of Au wt\% (the slope of the graph) of AuNP $1-\mathrm{V}$ is slightly higher than that of cationic AuNP 2-V. The Spacer molecule mainly determines the overall pattern, modulating the cellular uptake process. Whereas the surface charge on an AuNP determines the maximum $\mathrm{Au} w \mathrm{w} \%$ and the time to reach that point, possibly controlling the adsorption/desorption of the AuNPs with the cell.

As displayed in Fig. 2, there are size distributions of the designed AuNPs in this study. Size and surface modification of the polymeric nanomaterials are reported to be important for cellular uptake $e^{29,30}$. All the AuNPs designed in this study have Au core size of $20 \mathrm{~nm}$ in diameter. Compared with the charge-only AuNPs arranged as AuNP 1 and AuNP 2, because of the high molecular weight of the designed Spacers and introduced DOX, the hydrodynamic size $\left(\mathrm{D}_{\mathrm{H}}\right)$ of the Spacer-linked AuNPs measured in de-ionized water exhibits broad ranges from hundreds to thousands of $\mathrm{nm}$. Especially the bulky hydrophobic Spacer-linked AuNP 1-I and AuNP 2-I show high $\mathrm{D}_{\mathrm{H}}$ values than other Spacers. On the other hand, PEG 1000- and PEG 5000-conjugated AuNPs express relatively small differences. The $\mathrm{D}_{\mathrm{H}}$ of the AuNP is also strongly dominated by the designed Spacers, reflecting characteristic molecular interactions under an environmental condition.

Three-dimensional (3D) spatial distributions of the AuNPs in a cell. To visualize differentiated AuNP incorporation efficiency and their spatial distribution in a cell at the maximum AuNP uptake condition (the peak time in Fig. 2 and Fig. 3), consecutively recorded longitudinal depth profile images are obtained by twophoton microscopy (TPM) in Fig. 4 (also in Fig S5). The size of each image is $50 \mu \mathrm{m} \times 50 \mu \mathrm{m}$. A representative scale bar is on the image whose size is $10 \mu \mathrm{m}$. Four sets of the control images are arranged in Fig. 4a. The top line images of Fig. 4a are optical images color-scaled according to the signal intensity. The middle line images are obtained at the wavelengths shorter than $540 \mathrm{~nm}$ and green-colored against black background. Meanwhile, the bottom line images are obtained at the wavelengths longer than $540 \mathrm{~nm}$ and red-colored against black background. The images of AuNP 1 and AuNP 2 are detected exclusively at the wavelengths shorter than $540 \mathrm{~nm}$. Meanwhile, the A549 cells with and without DOX are exclusively captured at the wavelength longer than $540 \mathrm{~nm}$ by their autofluorescence (Supporting Information). Therefore, we confirm that controlled wavelength can reduce the autofluorescence of cancer cells and exclusively locate the AuNPs in a cell, maximizing the image quality.

Fig. $4 \mathrm{~b}$ and $4 \mathrm{c}$ display a series of designed Spacer-linked AuNPincorporated cell images. The upper line images in Fig. $4 \mathrm{~b}$ and $4 \mathrm{c}$ are AuNP-only images obtained at the wavelengths shorter than $540 \mathrm{~nm}$ (i). The cell-only images at longer than $540 \mathrm{~nm}$ wavelengths are redcolored against black background (ii). The lower row in Fig. $4 \mathrm{~b}$ and $4 \mathrm{c}$ display the combined images of (i) and (ii), to locate the AuNPs in a cell. In Fig. 4b, TPM images of the AuNP 1 series are arranged. AuNP 1-I is broadly dispersed throughout the cell, whereas the AuNP 1-II is rather concentrated at a specific depth of the cell (approximately $4 \mu \mathrm{m}$ from the surface). By contrast, PEG-conjugated AuNP 1-III and AuNP 1-IV do not have enough AuNPs that can be detected by TPM. The amphiphilic AuNP $1-\mathrm{V}$ is relatively well dispersed throughout the cell and is also intensively concentrated at a specific depth in a cell (between 2 and $4 \mu \mathrm{m}$ ). In contrast to the AuNP 1 series, all the AuNP 2 series in Fig. 4c have detectable AuNPs. Relatively higher amounts of AuNP 2-I and AuNP 2-II and relatively smaller amount of AuNP 2-III and AuNP 2-IV are concentrated in the specific depths of each cell. The amphiphilic AuNP 2-V is broadly dispersed throughout a cell with more intense concentration at a specific depth of a cell.

The obtained TPM images reflect the AuNP incorporation efficiency corresponding to the EDXS results. Each Spacer induces a spatial distribution of AuNPs in a cell characteristically. AuNPs having hydrophobic Spacers are effectively incorporated into the cells. Whereas the PEG-tethered Spacers are less effectively incorporated and/or localized in a cell. The amphiphilic Spacer generates broadly dispersed AuNPs in a cell and simultaneously concentrated at a specific layer of a cell.

Incorporation efficiency and spatial distribution of AuNPs in a cell at the initial stage (less than $\mathbf{3 0}$ min). To compare the designed AuNP interaction with the cell at fixed time duration, Au wt\% at 10, 20 and 30 min treatment obtained by the EDXS are graphically summarized in Fig. 5a. From the AuNP incorporation results at this relatively short time duration, the cellular interaction of the designed AuNPs at the initial stages are compared. Even for this short time duration, AuNP incorporation is characteristically differentiated by the Spacer molecules. The results are different from the fully-equilibrated Au wt\% compared in Fig. 3. AuNP 1-II displays prominently high incorporation in the cell at the given time. Overall, cationic AuNP 2 series exhibit relatively effective AuNP incorporation than anionic AuNP 1 series.

The representative zone-plate X-ray nanoscopy $(\mathrm{XN})$ images are displayed in Fig. 5b (Supporting Information). AuNP locations in a unit-cell scale are displayed at a fixed AuNP-treatment of $30 \mathrm{~min}$. $\mathrm{XN}$ is sensitive to detect small amount of AuNPs in a cell in-situ contrasting high X-ray absorption of AuNP versus X-ray transparency of cells, as well as phase contrast ${ }^{31,32}$. The dotted circles on each image highlight typical locations of the AuNPs in a cell. The XN image of AuNP 1-I is mainly located near the cell membrane. AuNP 1-II is highly incorporated inside the cell and also found at the membrane. Only a few AuNP 1-III and AuNP 1-IV are incorporated in the cells. AuNP 1-V is dispersed broadly inside the cell including the cytosol and the nucleus even though the amount is not much. For the cationic AuNP 2 series, the AuNP 2-I and AuNP 2-II are concentrated in the nucleus and also in the cell membrane. Majority of AuNP 2-III and AuNP 2-IV are trapped near the membrane and only some enter the cell. Most AuNP 2-V is concentrated in the nucleus.

In addition to the diversified $\mathrm{Au}$ wt $\%$ at the given time, the spatial distribution of the designed AuNPs in a cell is characteristic. Depending on the molecular structure of the Spacers conjugated to DOX and charges on AuNP surface, the incorporation pattern and the amount of AuNPs are diversified. At the initial stage of the AuNP incorporation occurring within 30 min-treatment, some AuNPs successfully reach deep into the cytosol and the nucleus. For both cationic and anionic AuNPs, hydrophobic and amphiphilic Spacers are advantageous for deep incorporation of AuNPs in a cell. With cationic surface charge of AuNP, PEG molecules generate a relatively strong interaction with the cell membrane, which allows AuNPs to be trapped in the cell membrane but not to be uptaken. Amphiphilic Spacers are advantageous for cellular uptake reaching nucleus.

In-vitro drug efficacy of the designed AuNPs. The in-vitro efficacy of DOX-tethered AuNPs is evaluated by fluorescence-activated cell sorting (FACS), discriminating dead to live cell ratio. The results of selected AuNP 1-V and AuNP 2-V are displayed in Fig. 6a together with the control systems of PEG 5000-only AuNP and free DOX. The 

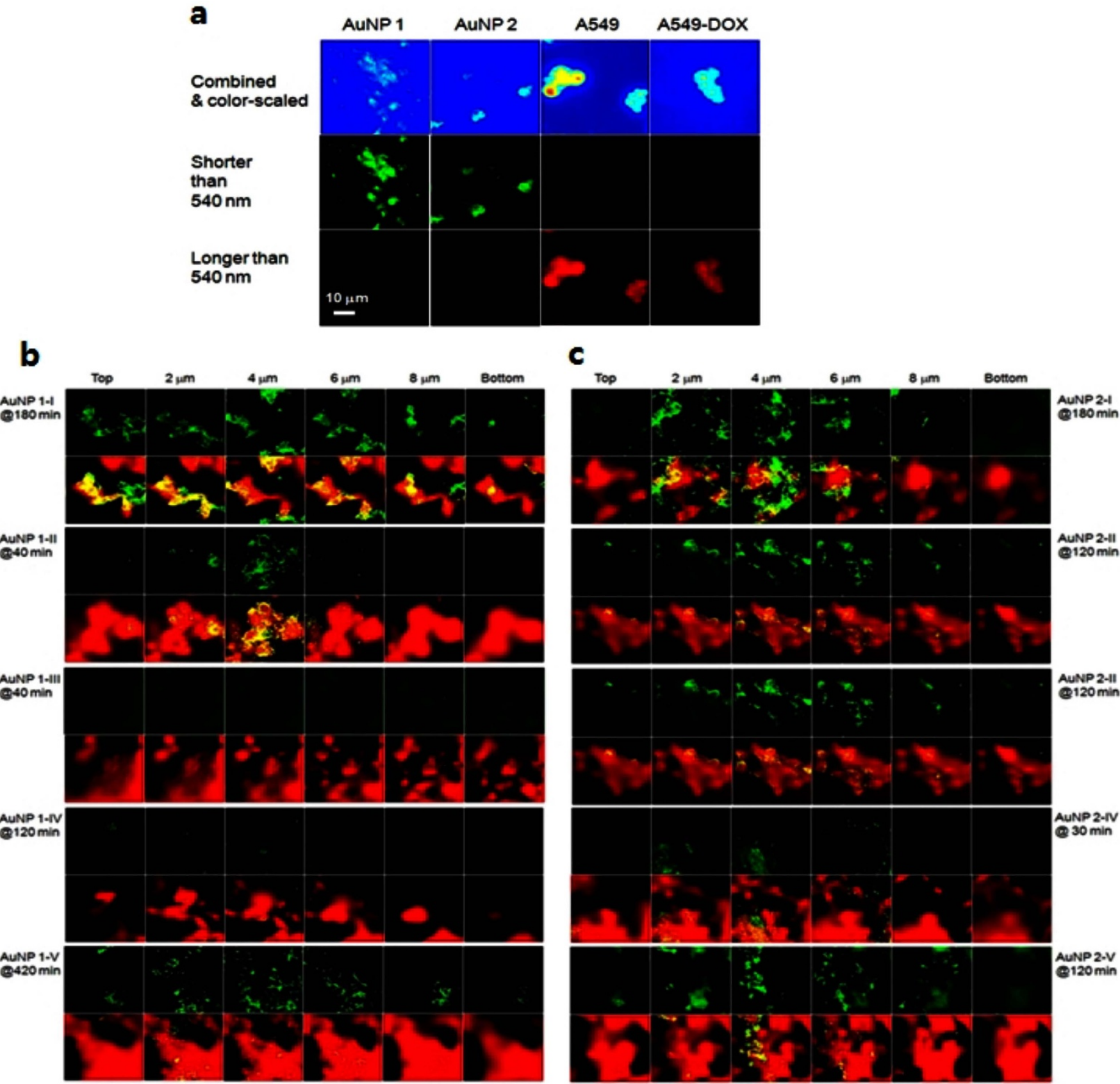

AUNP 2-11 (3) $120 \mathrm{~min}$

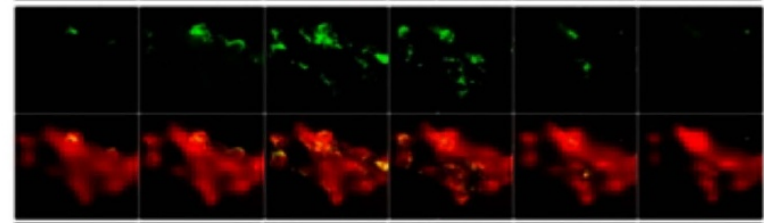

AuNP 2-11 A. $120 \mathrm{~min}$
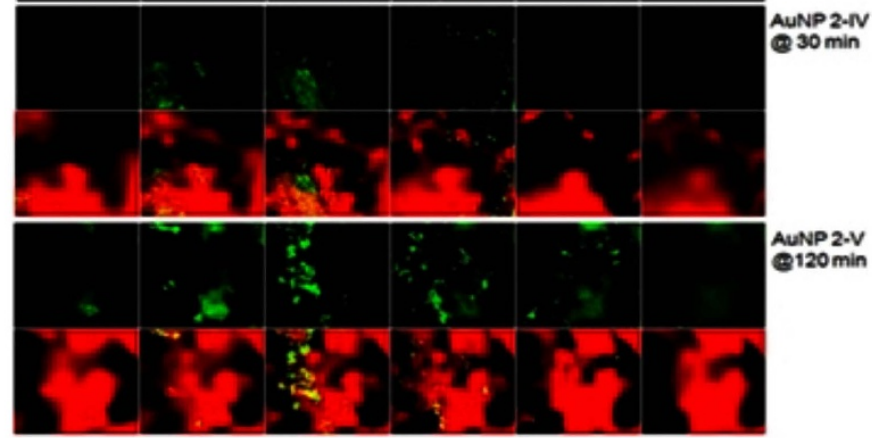

AUNP 2-V Q8120 $\mathrm{min}$

Figure $4 \mid$ Depth profiles images of a cell obtained by two-photon microscopy (TPM). From top to bottom of cell, $10 \mu \mathrm{m}$ depth is scanned.

The size of each image is $50 \mu \mathrm{m} \times 50 \mu \mathrm{m}$. (a) The images of anionic AuNP 1, cationic AuNP 2, A549 cell and DOX-treated A549 cells are arranged. The top line is the color-scaled optical microscopy images. The second line images are obtained by the wavelength shorter than $540 \mathrm{~nm}$, while the third lines are obtained by longer than $540 \mathrm{~nm}$. Series of images obtained for AuNP 1 series (b) and AuNP 2 series (c). The images are obtained at the time of the highest Au wt\% (from Fig. 3). The top line is the color-scaled optical microscopy images. The second line is the images obtained by the wavelength shorter than $540 \mathrm{~nm}$ (i), while the third is obtained by longer than $540 \mathrm{~nm}$ (ii). The middle line is the combined images of the first and the third (i + ii).

results of all the designed AuNPs are provided in Fig. S6. The cell viability of each system is examined at 10, 30, 60, and $120 \mathrm{~min}$. At $10 \mathrm{~min}$, all the systems are observed to have two types of cells due to the existence of live cells. The fluorescence intensity of the standard live cells are marked by a vertical dotted line in each graph, which is the positional baseline. The cell fluorescence intensity characteristically decreases by the contribution of the dead cells (wherein the AuNP effect is avoided; data not shown). The relatively high fluorescence intensity of PEG 5000-only AuNP reflects less toxicity to the cell. The intensity of free DOX decreases more significantly than that of the PEG 5000-only AuNPs, especially after 2 h. From $30 \mathrm{~min}$, the DOX-conjugated AuNP 1-V and AuNP 2-V exhibit a single peak with significantly decreased intensity lower than that of free DOX.

For clearer comparison, the normalized dead cell ratio is quantitatively provided in Fig. $6 \mathrm{~b}$. The dead cell ratio is estimated from the inverse value of the cell fluorescence intensity (Supporting Information). Based on the control systems, the dead cell ratio of the designed AuNPs becomes prominently high in a shorter time than that of free DOX. This result supports the hypothesis that designed AuNPs effectively deliver DOX penetrating cancer cells followed by rapid deployment of DOX. The whole procedure is more effective than the efficacy of free DOX. Depending on the Spacers, the dead cell ratio of both AuNP 1 and AuNP 2 becomes higher by the 

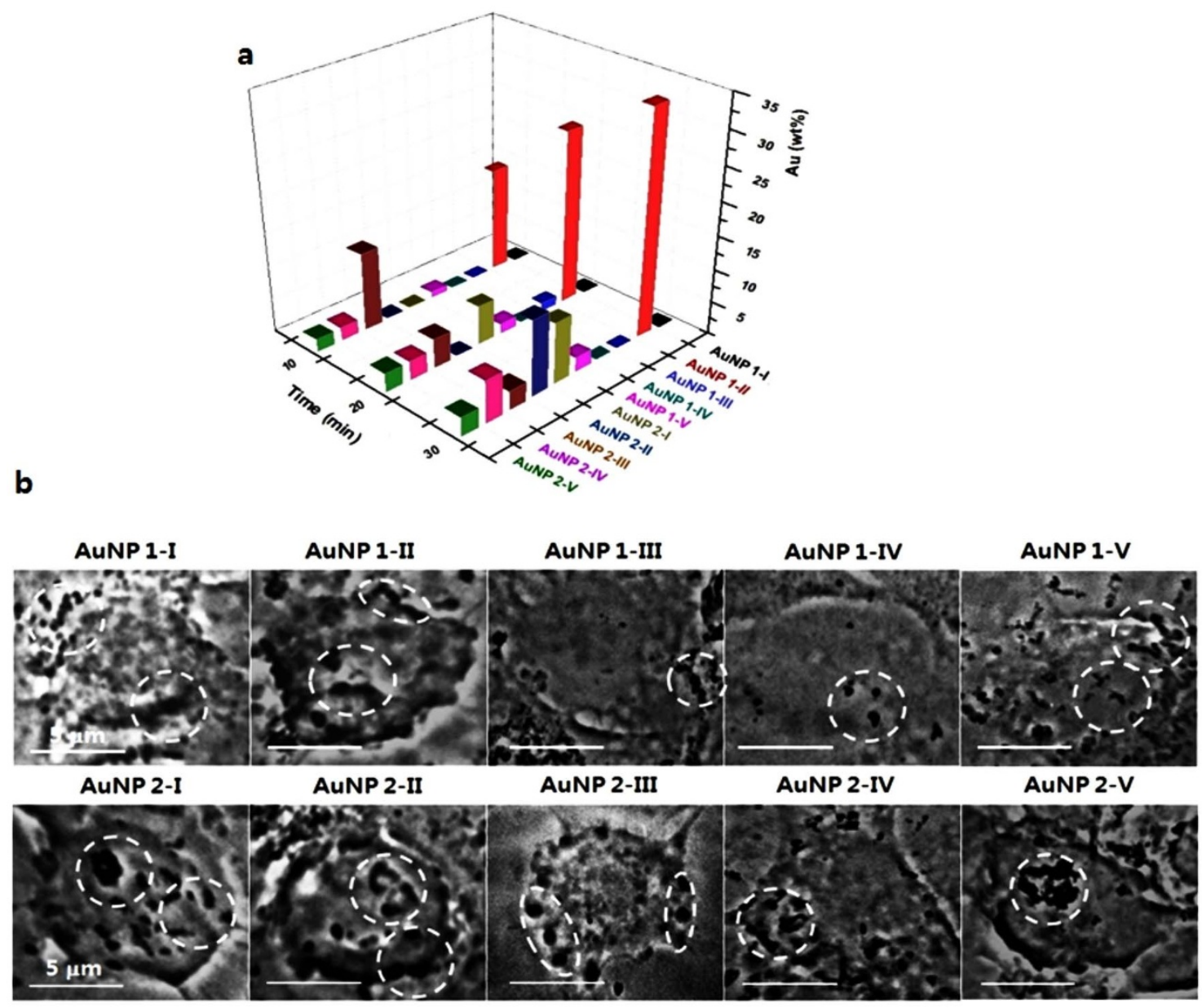

Figure $5 \mid$ (a) Au wt\% at 10, 20 and 30 min obtained by EDXS. (b) Zone-plate X-ray nanoscopy images obtained at $30 \mathrm{~min}$. The scale-bar is $5 \mu \mathrm{m}$.

sequence: Spacer V $>$ Spacer I (AuNP 2) \& II (AuNP 1) $>$ Spacer III $>$ Spacer IV.

Cellular uptake efficiency vs. in vitro drug efficacy. Fig. $6 \mathrm{c}$ compares the relation between the amount of incorporated AuNPs and the efficacy of the released DOX evaluated by EDXS and FACS as a function of time (reproduced from Fig. 3 and Fig. 6b). The designated time points are 30,60, and $120 \mathrm{~min}$, excluding the result of $10 \mathrm{~min}$ because of the dual distribution of the cells. The temporal variations of $\mathrm{Au} w \mathrm{w} \%$ obtained from the EDXS results (top graph) are fluctuated in some systems. However, the dead cell ratio obtained from the FACS results (bottom graph) increases with time in all the systems. By the increase in the DOX-conjugated AuNP incorporation, the detected $\mathrm{Au} w \mathrm{wt} \%$ and the dead cell ratios can be enhanced together. However, when the stepwise procedure of AuNP incorporation and the DOX deployment is complicated, Au wt $\%$ is not directly proportional to the dead cell ratio at the given time duration. For Spacer I, both the incorporated AuNPs (EDXS) and the dead cell ratio (FACS) increase together with time. The drug efficacy of AuNP 1-I seems to be more effective than AuNP 2-I, because AuNP 1-I shows higher dead cell ratio even with lower $\mathrm{Au}$ wt\% than that of AuNP 2-I. For Spacer II, AuNP 1-II initially exhibits high $\mathrm{Au} w \mathrm{w} \%$ and then decreases, while the dead cell ratio continues to increase. Therefore, the release of the DOX detached from the AuNP 1-II seems to be delayed compared with the AuNP incorporation rate into the cell. By contrast, AuNP 2-II continuously increases the dead cell ratio with time. This indicates similar release rate of DOX followed by its apoptotic efficacy with AuNP incorporation rate at the given time. PEG-conjugated AuNP 1-III increases the dead cell ratio with time, even with small amount of uptaken AuNPs. AuNP 2-III shows fluctuating Au wt\%, however the dead cell ratio continues to increase. Even though the interaction of PEGlinked AuNPs with the cell is unstable either by metabolic cycles of the cell itself or by the reversible adsorption/desorption procedure of NPs, DOX efficacy is detectable. The cellular uptake rate of PEGlinked AuNPs and DOX deployment might follow independent pathway. The AuNPs with Spacer IV display low dead cell ratios than that of Spacer III. This reflects that higher molecular weight PEG is not favorable for drug efficacy than that of lower molecular weight PEG, which might be caused from the increased stealth function. A slightly higher Au wt\% of AuNP 2-IV increases the dead cell ratio more effectively than AuNP 1-IV. The Au wt\% of Spacer V is low at the initial stage, but it significantly increases the dead cell ratio with time. This result indicates that efficacy of the released DOX is effectively fast. In terms of the time-dependent increase rate of the dead cell ratio (the slope of the graph), the amphiphilically combined Spacer V is most effective.

In-vivo drug efficacy in zebrafish cancer model. To evaluate in-vivo efficacy of the designed AuNPs, zebrafish (flkl: EGFP) model is employed. Compared with other vertebrate model system, zebrafish model provides amenability of in-vivo experiment and drug administration. Zebrafish possesses a complex circulatory system similar to that of mammals and an optical transparency which is beneficial for time-dependent in-vivo observation in in-situ condition. In addition, fast drug efficacy can be evaluated conveniently due to its fast metabolism and short life-cycle. Red fluorescencelabeled A549 treated with designed AuNPs, free DOX or PBS 

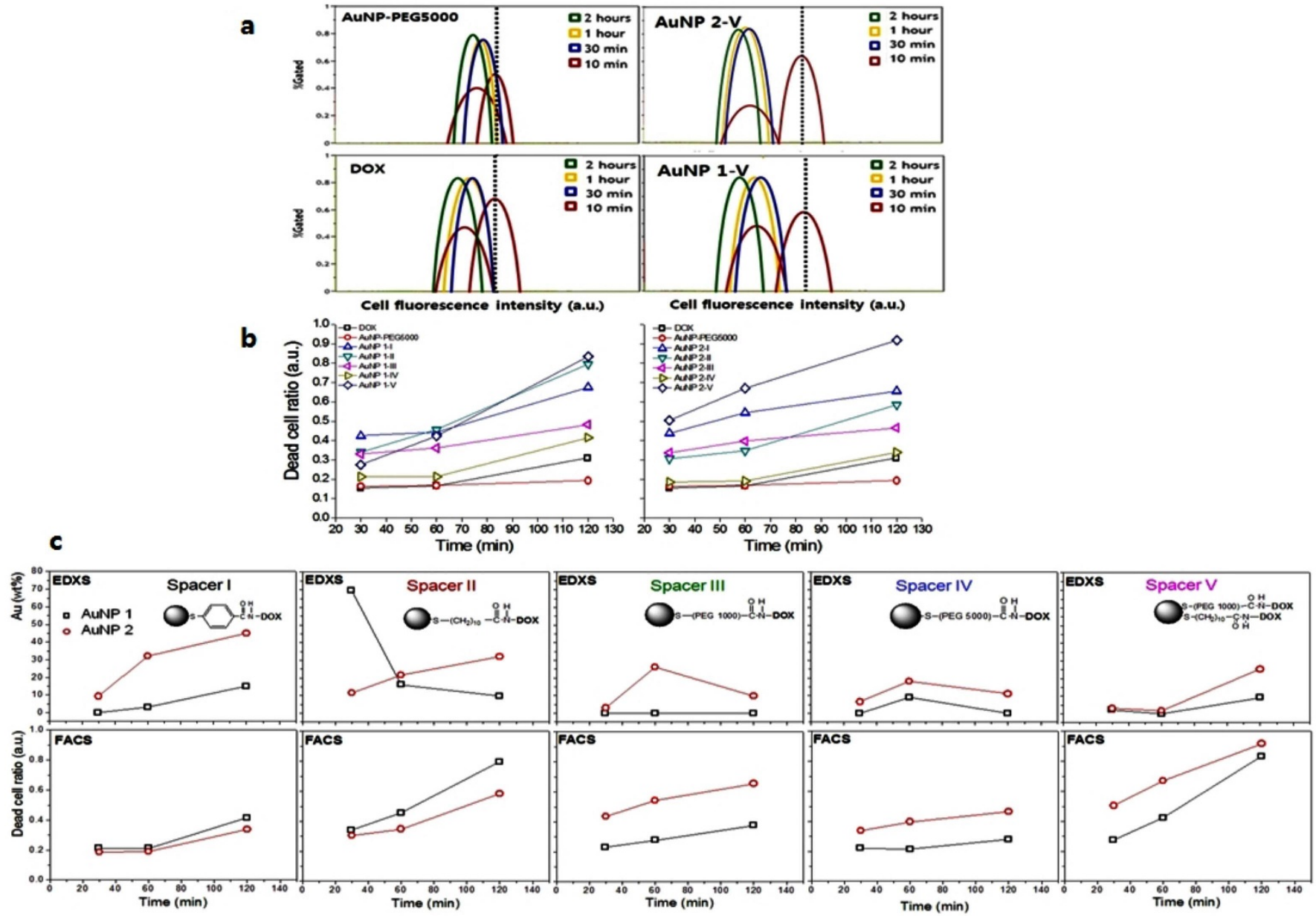

Cell fluorescence intensity (a.u.)

Figure $6 \mid$ (a) Fluorescence-activated cell sorting (FACS) results of PEG 5000-conjugated AuNP, free DOX and AuNP 1-V and 2-V. The results are obtained at $10 \mathrm{~min}, 30 \mathrm{~min}, 1 \mathrm{hr}$ and $2 \mathrm{hr}$. (b) Dead cell ratio changes with time. Free DOX and PEG 5000-conjugated AuNPs are compared as controls. (c) Au wt $\%$ vs. dead cell ratio of AuNPs for spacers I, II, III, IV and V at $30 \mathrm{~min}, 1 \mathrm{hr}$ and $2 \mathrm{hr}$.

solutions are injected (1000 cells/embryo) into the perivitelline space of yolk in $\mathrm{Tg}$ (flk1:EGFP) zebrafish embryos of four-day-postfertilization. The formed neoplasia is obviously protruded from abdomen of the zebrafish. Vessel formations are also investigated by green fluorescence (extracellular matrix, EC). After injection, the results of 24, 48 and $72 \mathrm{~h}$ are arranged for the selected AuNP $1-V$ system, free DOX and PBS solution (Fig. 7). Apparently, selected AuNP 1-V exhibits decreased tumor size detected by the decreased size and intensity of red fluorescence, which is especially prominent after $72 \mathrm{~h}$. Meanwhile, in case of free DOX and PBS solution, the region of the tumor becomes larger or spreads into other organs as time goes on. In addition, the designed AuNP 1-V does not inhibit the neo-vessel formation which is one of the typical anticancer mechanisms. Therefore, the designed AuNP specifically enhances the apoptotic effect of the delivering drug, DOX employed in this study.

\section{Discussion}

Our study demonstrates that high-resolution profiles of drugconjugated NP distribution in single cells in a model tumor using $\mathrm{X}$-ray nanoscopy and two-photon microscopy. Moreover, quantitative data can be either modeled to predict cumulative drug effects which can be further extrapolated to human settings to provide insights into drug action previously undetectable. The findings with drug-conjugated AuNPs in the present study demonstrate ubiquitous distribution of drugs virtually all cells in a tumor, rapid permeation to the nucleus with effectively designed Spacer molecules. There are a number of different models describing drug distribution and responses ${ }^{33}$. Although these models have been developed to incorporate delivery limitations or more complex pharmacodynamic measures, there is currently no method that incorporates the known cellto-cell variability in cancer phenotypes, the heterogeneous nature of drug delivery and the variability of cellular responses. To reconcile the gap between the reported models and molecular pathways at the cellular level, we have related the single-cell-level in-vitro nanotherapeutics incorporation and its apoptotic action as well as drug efficacy in in-vivo tumor model. That is capable of providing insight into drug deployment from the nanoptherapeutics and response relationships at the single cell level further extending to responsiveness in collective tumor tissues. Few studies have been systematically elucidated on the relation between cellular interaction of the nanotherapeutics and the actual drug efficacy. Connecting the in-vitro and invivo results observed in this study, it is suggested that fast cellular uptake does not directly induce the fast effect of apoptotic drugs.

In terms of the molecular structure of the designed nanotherapeutics, the result validates that Spacer molecules are critically important to determine the AuNP behaviors in cancer cells in-vitro and in-vivo: the molecular structure of the Spacer molecule dominates the timedependent cellular uptake pattern and spatial arrangement of AuNPs in a single cell and further their drug efficacy. Especially, the designed drug-conjugated NPs exhibit effective cellular uptake and faster drug efficacy compared with free drugs. By the effective cellular uptake procedure, the NPs are homogeneously distributed in the cells, reaching deep into the cytosol with a high loading efficiency. On 


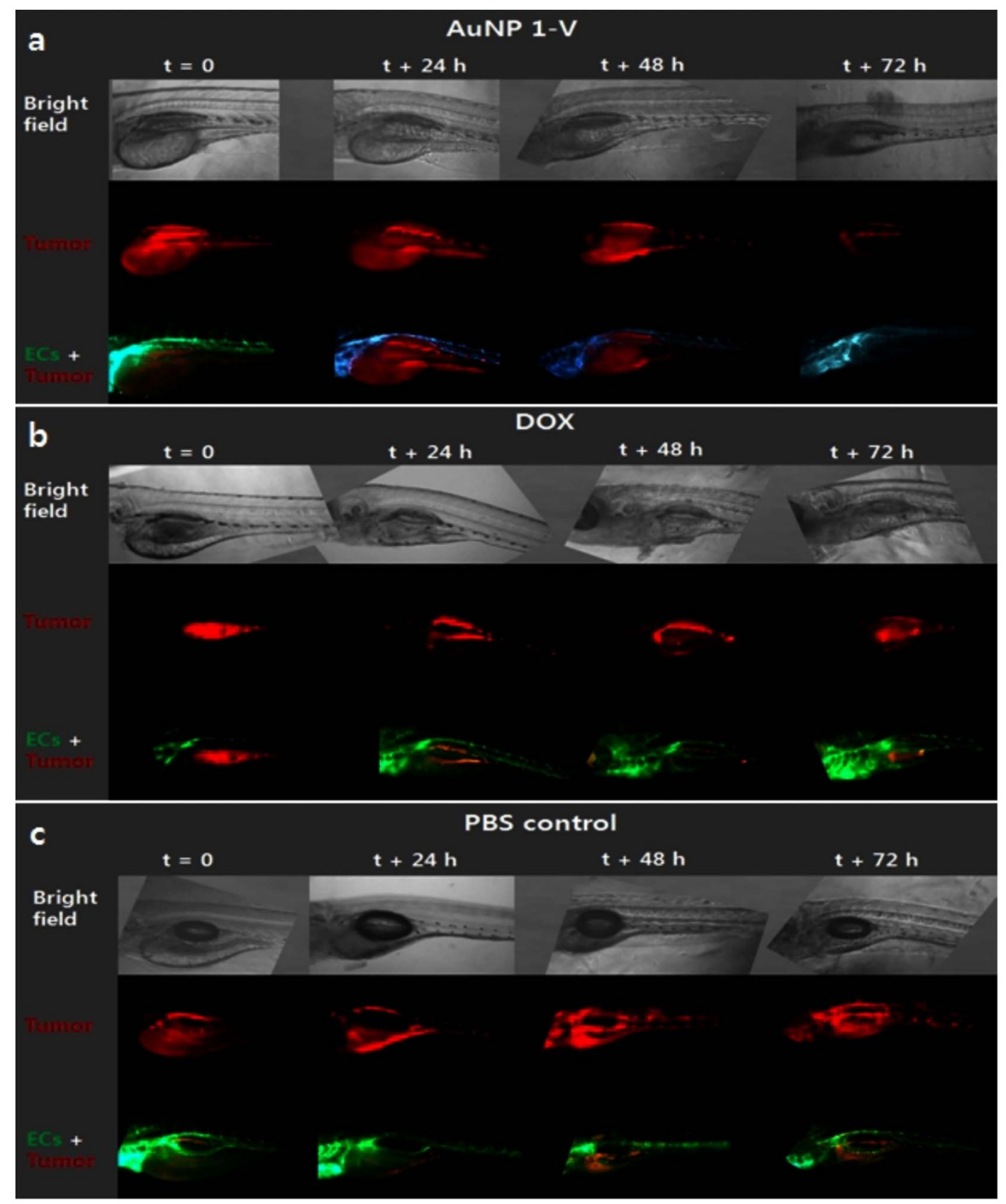

Figure $7 \mid$ In-vivo tumor xenografts in zebrafish embryos treated with (a) AuNP 1-V, (b) free DOX and (c) PBS control.

the other hand, less efficient molecular design of the Spacers allows AuNPs (if there is any uptake) in the endosome or near the cell membrane with a low loading efficiency. With the designed Spacer molecules tethering drugs on the NPs, the cellular uptake process and the drug release/efficacy are diversified. Hydrophobic spacers are advantageous for an effective NP uptake followed by drug release at the short time duration. On the other hand, amphiphilically composed Spacers are beneficial for the continuous incorporation of NPs with time as well as high dead cell ratio per unit time. The magnitude and evolution of drug gradients are typically measured and subsequently modeled using imaging drugs. In this aspect, studies utilizing drug-conjugated NPs can be more versatile than conventional expectations. The results of drug-conjugated NPs can also be extrapolated to various other drug and drug candidates based on concentrationtime profiles. Using image quantification based on the detected $\mathrm{Au}$ element, we find that intracellular drug concentrations will reach sufficiently high levels in cancer cells. Measuring drug concentrations by X-ray imaging at the single-cell level could be further expanded to other drug classes for example, various chemical therapeutics, siRNA, antibodies, proteins, other engineered cells.

Therefore, we confirm that NP-based molecular transport into a cell can be developed with a great potential to reduce yet-resolved adverse effects of anticancer drugs. We also suggest that this technology can be further developed for novel nanotherapeutics design and various NP-mediate therapeutic technologies.

\section{Methods}

DOX-tethered surface-modified AuNPs. The average core size obtained by transmission electron microscopy (TEM) is approximately $20 \mathrm{~nm}$ for all the AuNPs. The concentration of standard AuNP stock solution $(\times 1)$ is controlled at $1 \mathrm{mM}$ of Au (Supporting Information). The concentration of the standard AuNP solution is $2.4 \times 10^{12} \mathrm{AuNP} / \mathrm{mL}$. Then the number of DOX per unit AuNP is controlled as $1.15 \times 10^{3} \mathrm{DOX} / \mathrm{AuNP}$. The carboxyl groups of the designed spacers are activated by 
$N$-hydroxysuccinimide and $N, N^{\prime}$-dicyclohexylcarbodiimide. The activated acid is then reacted with the amine group of DOX. All the reaction mixtures are dialyzed against Milli-Q water for purification. For respective AuNP, the spacers containing $0.05 \mathrm{mg}$ DOX $\left(\mathrm{M}_{\mathrm{w}}: 543.52,9.2 \times 10^{-8}\right.$ mole DOX, $5.5 \times 10^{16}$ DOX molecules) are introduced to a $20 \mathrm{~mL}$ standard citrate-covered AuNP stock solution $\left(1 \mathrm{mmol} \cdot \mathrm{L}^{-1}\right.$ of $\mathrm{Au})$. Then, the number of DOX per unit AuNP is controlled as $1.15 \times 10^{3} \mathrm{DOX} /$ AuNP. The completion of the reaction is confirmed by the absence of the thiolcontaining DOX-conjugated spacers in the reaction solvent (confirmed by UV-vis spectrum and fluorescence, data is not shown). To the aforementioned DOXconjugated AuNPs, negatively and positively charged thiol ligands are further introduced to modify the surface properties of the DOX-conjugated AuNPs: thioglycolic acid $\left(\mathrm{SH}-\mathrm{CH}_{2} \mathrm{COOH}\right.$, designated as AuNP 1) and 6-thioguanine (SH- $\mathrm{C}_{5} \mathrm{H}_{4} \mathrm{~N}_{5}$, designated as AuNP 2). The obtained AuNP solutions are purified by dialysis for more than 2 days. The detailed procedures are provided in Supporting Information.

Cell preparation. A549 cells are cultured in RPMI-1640 medium containing 10\% fetal bovine serum and $1 \%$ penicillin-streptomycin. A low-serum growth supplement containing $20 \%$ fetal bovine serum and $1 \%$ penicillin-streptomycin is also added. The cells are cultured in a humidified atmosphere with $5 \% \mathrm{CO}_{2}$ at $37^{\circ} \mathrm{C}$. A trypsin (0.25\%)-EDTA solution is used to detach the cells from the culture flask. Detailed procedures are in Supporting Information.

SEM-EDXS. The cells are loaded onto a cover glass and then cultured for $24 \mathrm{~h}$ Thereafter, the cells are treated with a series of surface-modified AuNPs for the designated time. The samples are fixed with $2.5 \%$ glutaraldehyde in Dulbecco's phosphate buffered saline for $20 \mathrm{~min}$ at room temperature, washed thrice with deionized water, and then air-dried. The samples are Ni-coated using a coater (Quorum Technology, SC7640 model). SEM images are captured using an XL30SFEG (Philips) SEM connected to an EDXS system at an acceleration voltage of $20 \mathrm{kV}$. SEM and EDXS measurements are performed using a XL30SFEG electron probe microanalyzer equipped with a Genesis System EDAX detector. The detailed procedures are described in Supporting Information.

TPM. An upright microscope (BX51; Olympus) is used to perform TPM. The system is equipped with a Ti:sapphire femtosecond laser (Chameleon; Coherent) with a tuning range of $680 \mathrm{~nm}$ to $1020 \mathrm{~nm}$. To generate the two-photon effect, the laser source is operated at $80 \mathrm{MHz}$ repetition rate and $140 \mathrm{fs}$ pulse width. The beam first passes through a half-wave plate and then through a polarizer for power control. The beam is expanded twice by passing it through a pair of plano-convex lenses, and then the expanded and excited light are passed through a resonant $x-y$ scanner (counter rotation scanner, GSI Lumonics) operating at $8 \mathrm{KHz}$ scanning speed. Details are provided in Supporting Information.

Zebrafish preparation and cancer cell microinjection. Zebrafish is maintained in $\mathrm{E} 3$ embryo media $\left(15 \mathrm{mM} \mathrm{NaCl}, 0.5 \mathrm{mM} \mathrm{KCl}, 1 \mathrm{mM} \mathrm{MgSO}_{4}, 1 \mathrm{mM} \mathrm{CaCl}_{2}, 0.15 \mathrm{mM}\right.$ $\mathrm{KH}_{2} \mathrm{PO}_{4}, 0.05 \mathrm{mM} \mathrm{Na}_{2} \mathrm{HPO}_{4}, 0.7 \mathrm{mM} \mathrm{NaHCO}, 10-5 \%$ methylene blue; $\mathrm{pH} 7.4$ ) containing 1-phenyl 2-thiourea (Sigma-Aldrich) to prevent pigmentation at $29^{\circ} \mathrm{C}$. Red fluorescence labeled cancer cell pellet and matrigel (BD Biosciences) are mixed with $1 / 1(\mathrm{vol} / \mathrm{vol})$ at $4{ }^{\circ} \mathrm{C}$. To this call-gel mixture, doxorubicin or doxorubicintethered surface-modified AuNP solutions are mixed with 3/1 (vol/vol). This cell suspension mixture containing 1000 cells is loaded into a borosilicate glass needle and implanted into each zebrafish of 4 day-post-fertilization embryo through the yolk in a single injection by using an electronically regulated air-pressure microinjector (Eppendorf, FemtoJet express). After injection, zebrafish is washed once with E3 embryo media and examined the presence of fluorescent cells. Forty zebrafishes are selected and transferred to 24-well plate containing $1 \mathrm{~mL}$ of fresh E3 embryo media for photography. E3 embryo media is changed daily and maintained under normal fish husbandry conditions for 3 days. For the work with zebrafish, all the experiments were performed in accordance with relevant guidelines and regulations.

1. Davis, M. E., Chen, Z. G. \& Shin, D. M. Nanoparticle therapeutics: An emerging treatment modality for cancer. Nat Rev Drug Discov 7, 771-782 (2008).

2. Petros, R. A. \& DeSimone, J. M. Strategies in the design of nanoparticles for therapeutic applications. Nat. Rev. Drug Discov. 9, 615-627 (2010).

3. Chen, Y., Bathula, S. R., Yang, Q. \& Huang, L. Targeted nanoparticles deliver siRNA to melanoma. J. Invest. Dermatol. 130, 2790-2798 (2010).

4. Herbig, M. E., Assi, F., Textor, M. \& Merkle, H. P. The cell penetrating peptides pVEC and W2-pVEC induce transformation of gel phase domains in phospholipid bilayers without affecting their integrity. Biochemistry 45, 3598-3609 (2006)

5. Takeuchi, T. et al. Direct and rapid cytosolic delivery using cell-penetrating peptides mediated by pyrenebutyrate. ACS Chem. Biol. 1, 299-303 (2006).

6. Nativo, P., Prior, I. A. \& Brust, M. Uptake and intracellular fate of surfacemodified gold nanoparticles. ACS Nano 2, 1639-1644 (2008).

7. Pernodet, N. et al. Adverse effects of citrate/gold nanoparticles on human dermal fibroblasts. Small 2, 766-773 (2006).

8. Wang, F. et al. Doxorubicin-tethered responsive gold nanoparticles facilitate intracellular drug delivery for overcoming multidrug resistance in cancer cells. ACS Nano 5, 3679-3692 (2011).
9. Schluep, T. et al. Pharmacokinetics and biodistribution of the camptohecinpolymer conjugate IT-101 in rats and tumor-bearing mice. Cancer Chemother. Pharmacol. 57, 654-662 (2006).

10. Matsumura, Y. \& Maeda, H. A new concept for macromolecular therapeutics in cancer chemotherapy: Mechanism of tumoritropic accumulation of proteins and the antitumor agent SMANCS. Cancer Res. 46, 6387-6392 (1986).

11. Dreher, M. R. et al. Tumor vascular permeability, accumulation, and penetration of macromolecular drug carriers. J. Natl. Cancer Inst. 98, 335-344 (2006).

12. Gardner, E. R. et al. Randomized crossover pharmacokinetic study of solventbased paclitaxel and nab-paclitaxel. Clin. Cancer Res. 14, 4200-4205 (2008).

13. Hu, Y., Xie, J. W., Tong, Y. W. \& Wang, C. H. Effect of PEG conformation and particle size on the cellular uptake efficiency of nanoparticles with the HepG2 cells. J. Controlled Release 118, 7-17 (2007).

14. Sahay, G., Alakhova, D. Y. \& Kabanov, A. V. Endocytosis of Nanomedicines. J. Controlled Release 145, 182-195 (2010).

15. Chen, M. Y. et al. Surface properties, more than size, limiting convective distribution of virus-sized particles and viruses in the central nervous system. J. Neurosurg. 103, 311-319 (2005).

16. Nel, A. E. et al. Understanding biophysicochemical interactions at the nano-bio interface. Nat. Mater. 8, 543-557 (2009).

17. Chithrani, B. D. \& Chan, W. C. W. Elucidating the mechanism of cellular uptake and removal of protein-coated gold nanoparticles of different sizes and shapes. Nano Lett. 7, 1542-1550 (2007).

18. Xie, C., Xu, N., Kohler, Y. \& Hou, S. Controlled PEGylation of monodisperse $\mathrm{Fe} 3 \mathrm{O} 4$ nanoparticles for reduced non-specific uptake by macrophage cells. $A d v$. Mater. 19, 3163-3166 (2007).

19. Chang, E., Yu, W. W., Colvin, V. L. \& Drezek, R. Quantifying the influence of surface coatings on quantum dot uptake in cells. J. Biomed. Nanotechnol. 1 , 397-401 (2005).

20. Alberts, A. J. B., Lewis, J., Raff, M., Roberts, K. \& Walter, P. Molecular Biology of the Cell, Garland Science, Taylor and Francis Group New York, 2002.

21. Harush-Frenkel, O., Debotton, N., Benita, S. \& Altschuler, Y. Targeting of nanoparticles to the clathrin-mediated endocytic pathway. Biochem. Biophys. Res. Commun. 353, 26-32 (2007).

22. Cho, E. C., Xie, J. W., Wurm, P. A. \& Xia, Y. N. Understanding the role of surface charges in cellular adsorption versus internalization by selectively removing gold nanoparticles on the cell surface with $\mathrm{I}_{2} / \mathrm{KI}$ etchant. Nano Lett. 9, 1080-1084 (2009).

23. Wilhelm, C. et al. F. Intracellular uptake of anionic superparamagnetic nanoparticles as a function of their surface coating. Biomaterials 24, 1001-1011 (2003).

24. Shi, X. Y., Thomas, T. P., Myc, L. A., Kotlyar, A. \& Baker, J. R. Synthesis, characterization, and intracellular uptake of carboxyl-terminated poly(amidoamine) dendrimer-stabilized iron oxide nanoparticles. Phys. Chem. Chem. Phys. 9, 5712-5720 (2007).

25. Patil, S., Sandberg, A., Heckert, E., Self, W. \& Seal, S. Protein adsorption and cellular uptake of cerium oxide nanoparticles as a function of zeta potential. Biomaterials 28, 4600-4607 (2007).

26. Mortensen, L. J., Oberdorster, G., Pentland, A. P. \& DeLouise, L. A. In vivo skin penetration of quantum dot nanopartiles in the murine model: The effect of UVR. Nano Lett. 8, 2779-2787 (2008).

27. Hillaireau, H. \& Couvreur, P. Nanocarriers' entry into the cell: relevance to drug delivery. Cell. Mol. Life Sci. 66, 2873-2896 (2009).

28. Hsu, Y.-L., Kuo, P.-L. \& Lin, C.-C. Proliferative inhibition, cell-cycle dysregulation, and induction of apoptosis by ursolic acid in human non-small cell lung cancer A549 cells. Life Sciences 75, 2303-2316 (2004).

29. Zhu, J. et al. Size-dependent cellular uptake efficiency, mechanism, and cytotoxicity of silica nanoparticles toward HeLa cells. Talanta 107, 408-415 (2013).

30. He, C., Hu, Y., Yin, L., Tang, C. \& Yin, C. Effects of particle size and surface charge on cellular uptake and biodistribution of polymeric nanoparticles. Biomaterials 31, 3657-3666 (2010).

31. Ahn, S., Jung, S. Y., Seo, E. \& Lee, S. J. Gold nanoparticle-incorporated human red blood cells (RBCs) for X-ray dynamic imaging. Biomaterials 32, 7191-7199 (2011).

32. Ahn, S., Jung, S. Y., Lee, J. P., Kim, H. K. \& Lee, S. J. Gold Nanoparticle Flow Sensors Designed for Dynamic X-ray Imaging in Biofluids. ACS Nano 4, 3753-3762 (2010).

33. Sun, H. et al. Population pharmacokinetics. A regulatory perspective. Clin. Pharmacokinet. 37, 41-58 (1999).

\section{Acknowledgements}

This work was supported by the Creative Research Initiatives (Diagnosis of Biofluid Flow Phenomena and Biomimic Research) of the Ministry of Education, Science and Technology (MEST) and the National Science Foundation of Korea. This research was jointly supported by the World Class University program funded by MEST (R31-2008-000-10105-0). The authors are grateful for the valuable help in the X-ray imaging experiments performed at the 1B2 and 7B2 beamlines of the Pohang Accelerator Laboratory (Pohang, Korea). 


\section{Author contributions}

S.A., K.K., S.J.L. developed the concepts and designed the experiments; S.A. and E.S. performed the experiments; S.A., E.S. analyzed the results and wrote the paper.

\section{Additional information}

Supplementary information accompanies this paper at http://www.nature.com/ scientificreports
Competing financial interests: The authors declare no competing financial interests.

How to cite this article: Ahn, S., Seo, E., Kim, K. \& Lee, S.J. Controlled cellular uptake and drug efficacy of nanotherapeutics. Sci. Rep. 3, 1997; DOI:10.1038/srep01997 (2013).

(c) (i) (2) This work is licensed under a Creative Commons Attributioncc. ${ }_{\mathrm{BY}} \mathrm{NC}$ SA NonCommercial-ShareAlike 3.0 Unported license. To view a copy of this license, visit http://creativecommons.org/licenses/by-nc-sa/3.0 